\title{
Fauna flebotomínica (Díptera, Psychodidae, Phlebotominae) do Estado Bolivar, Venezuela
}

\author{
Sand-fly fauna (Díptera, Psychodidae, Phlebotominae) \\ in Bolivar State, Venezuela
}

Ricardo González e Rodolfo Devera

\begin{abstract}
Resumo Foi feito um estudo para conhecer a fauna flebotomínica do Estado Bolívar, Venezuela. Foram identificados 2364 flebotomíneos distribuídos em 18 espécies. As espécies que podem estar participando da transmissão da Leishmaniose tegumentar americana no Estado Bolívar são possivelmente Lutzomyia anduzei, L. wellcomei e L. squamiventris ao Sul do estado, e L. flaviscutellata e L. gomezi as espécies transmissoras ao Norte.
\end{abstract}

Palavras-chaves: Leishmaniose tegumentar americana. Flebotomíneos. Lutzomyia. Venezuela.

Abstract $A$ descriptive study is presented of sand-fly fauna in Bolivar State, Venezuela. Identification was made of 2,364 sand-flies belonging to 18 species. The vectors of American cutaneous leishmaniasis in the different geographic regions of Bolivar State are possibly L. anduzei, $\mathrm{L}$. wellcomei and L. squamiventris in the South, and L. flaviscutellata and L. gomezi in the North. Key-words: American cutaneous leishmaniasis. Sand-flies. Lutzomyia. Venezuela.

Leishmaniose tegumentar americana (LTA) é causa importante de morbidade na Venezuela e em especial no Estado Bolívar onde tem um caráter ocupacional. Neste estado, a doença está associada à atividade mineira, fundamentalmente extração de ouro e diamantes, e com as atividades agrícolas ${ }^{5}$.

São poucas as informações que existem sobre a epidemiologia da LTA no Estado Bolívar, em particular no que diz respeito a seus transmissores. Somente se conhece a presença de algumas espécies de flebotomíneos, sem saber qual é sua importância vetorial ${ }^{13}$.
Entre dezembro de 1993 e junho de 1996, realizou-se um inquérito entomológico para registrar a fauna flebotomínica em diferentes localidades do Estado Bolívar, determinar as espécies antropofílicas dominantes e estudar a distribuição geográfica e alguns aspectos ecológicos dos flebotomíneos no Estado Bolívar.

As áreas estudadas eram habitadas por cinco populações humanas do Estado Bolívar, sendo que, quatro eram comunidades agrícolas: Maripa, Aripao, El Miamo e San José de Hacha, localizadas ao norte do Estado Bolívar. A quinta localidade estudada foi Icabaru; os habitantes

\footnotetext{
Laboratorio de Vectores de Enfermedades Tropicales. Unidad de Estudios Básicos e Departamento de Parasitología y Microbiología. Universidad de Oriente, Núcleo Bolívar, Venezuela.

Suporte financeiro: Fundacite-Guayana e Consejo de Investigación de la Universidade de Oriente.

Endereço para correspondência: Prof. Ricardo González. Laboratorio de Vectores de Enfermedades Tropicales/Unidad de Estudios Básicos/Universidad de Oriente. Núcleo Bolívar. Calle San Simón, Apto Correo 527, Ciudad Bolívar, Venezuela.

Telefax: 005885312585

E-mail: ricardog@telcel.net.ve

Recebido para publicação em 27/10/98.
} 
realizavam atividades de mineração, e estava localizada ao sul a 30 quilômetros da fronteira com o Brasil. Dessas localidades, somente em San José de Hacha e Icabaru havia focos ativos de LTA.

Os flebotomíneos foram capturados manualmente com tubo de aspiração, utilizando armadilha luminosa de Shannon, isca humana e busca ativa nos refúgios naturais. No laboratório os flebotomíneos foram dissecados e montados em solução de Berlese. Para sua identificação utilizaram-se as chaves de Theodor ${ }^{6}$, Forattini ${ }^{2}$, Feliciangeli ${ }^{1}$ e Young e Duncan ${ }^{8}$. Capturou-se um total de 2.387 exemplares, 1.475 fêmeas $(61,8 \%)$ e 912 machos (33,2\%). Foram identificados 2.364 flebotomíneos distribuídos em 18 espécies. Outros 33 exemplares ainda não foram identificados adequadamente. Na Tabela 1, registra-se o total geral de flebotomíneos identificados, observa-se que as espécies mais abundantes foram Lutzomyia anduzei(61,1\%), L. amazonensis (19\%), L. squamiventris $(8,8 \%)$ L. antunesi $(2,4 \%)$, L. trinidadensis $(2 \%)$ e L. wellcomei $(1,8 \%)$. Das 18 espécies capturadas, oito não haviam sido ainda registradas no Estado Bolívar ${ }^{13}$.

Das espécies capturadas, nove são antropofílicas, tendo diferenças segundo a localidade estudada. Ao sul, as espécies antropofílicas mais abundantes foram $L$. amazonensis, $L$. anduzei, $L$. squamiventris e L. wellcomei; enquanto que ao norte L. gomezi foi a espécie antropofílica mais importante.

No Icabaru, onde existe atualmente um surto de LTA, capturou-se o maior número de flebotomíneos. Nenhum foi capturado nos abrigos naturais, apesar de terem sido feitas sete capturas na localidade. Em três das outras zonas

Tabela 1 - Fauna flebotomínica em 5 localidades do Estado Bolívar, Venezuela.

\begin{tabular}{|c|c|c|c|c|}
\hline Espécies & Fêmeas & Machos & Total & $\%$ \\
\hline L. anduzei & 622 & 822 & 1444 & 61,1 \\
\hline L. amazonensis* & 449 & 0 & 449 & 19,0 \\
\hline L. squamiventris & 206 & 1 & 207 & 8,8 \\
\hline L. antunesi ${ }^{\star}$ & 28 & 28 & 56 & 2,4 \\
\hline L. trinidadensis ${ }^{*}$ & 24 & 24 & 48 & 2,0 \\
\hline L. wellcomei $i^{* *}$ & 43 & 0 & 43 & 1,8 \\
\hline L. flaviscutellata & 37 & 0 & 37 & 1,6 \\
\hline L. gomezi & 18 & 15 & 33 & 1,4 \\
\hline L. dendrophyla & 0 & 14 & 14 & 0,6 \\
\hline L. shannoni & 8 & 2 & 10 & 0,4 \\
\hline L. auraensis & 5 & 2 & 7 & 0,3 \\
\hline L. davisi & 4 & 0 & 4 & 0,2 \\
\hline L. ovallesi* & 3 & 1 & 4 & 0,2 \\
\hline L. punctigeniculata* & 3 & 0 & 3 & 0,1 \\
\hline L. spinosa & 2 & 0 & 2 & 0,1 \\
\hline L. bettinii ${ }^{*}$ & 1 & 0 & 1 & 0,0 \\
\hline L. infraspinosa* & 0 & 1 & 1 & 0,0 \\
\hline L. panamensis & 1 & 0 & 1 & 0,0 \\
\hline Total: 18 espécies & 1454 & 910 & 2364 & 100,0 \\
\hline
\end{tabular}

* Novo registro para o Estado Bolívar.

** Novo registro para Venezuela.

estudadas, localizadas ao norte, foram capturados exemplares nos refúgios naturais.

Também no Icabaru, foram dissecados e examinados os tratos digestivos de 142 flebotomíneos das seguintes espécies: $L$. anduzei (83,1\%), L. amazonensis (7,1\%), L. squamiventris $(2,8 \%)$, L. shannoni $(2,1 \%)$, L. wellcomei $(2,1 \%)$,
L. flaviscutellata $(1,4 \%)$ e $L$. antunesi $(1,4 \%)$. Todos resultaram negativos para promastigotas de Leishmania.

Na América, L. anduzei, L. wellcomei, L. squamiventris, L. gomezi e L. flaviscutellata tem sido assinaladas como espécies vetoras de $\operatorname{LTA}^{4}{ }^{7}$. No foco de Icabaru, por sua abundância 
e hábitos antropifílicos preponderantes, possivelmente $L$. anduzei, L. wellcomei e L. squamiventris, sejam os vetores principais, embora não se tenha encontrado infecção natural dessas espécies em nosso estudo. No outro foco ativo de LTA em San José de Hacha, L. gomezi é a espécie mais antropofílica e abundante, sendo possivelmente a vetora primária da doença. Nas demais localidades estudadas, embora não se tinha registrado casos de $\mathrm{LTA}$, existem espécies de flebotomíneos como $L$. gomezie $L$. flaviscutellata de reconhecida capacidade vetorial no novo mundo ${ }^{147}$.

As espécies mais abundantes de Lutzomyia, identificadas ao sul do estado, foram diferentes das capturadas nas localidades do norte, o que sugere diferenças na ecologia e distribuição geográfica dos flebotomíneos no Estado Bolívar.

\section{REFERÊNCIAS BIBLIOGRÁFICAS}

1. Feliciangeli MD. La fauna flebotómica (Díptera: Psychodidae) en Venezuela: I. Taxonomía y distribución geográfica. Boletín de la Dirección de Malariología y Saneamiento Ambiental 28:99-113, 1988.

2. Forattini OP. Entomología Médica. Ed. Edgard Blucher Ltda. São Paulo, Brasil, 1973.

3. González R. Estudio de la fauna flebotómica del estado Bolívar - La Paragua, Distrito Heres. Acta Científica Venezolana 35 (supl 1): 384, 1984.

4. Grimaldi Jr G, Testh RB. Leishmaniasis of the new world. Clinical Microbiology Reviews 6: 230-250, 1993.

5. Navas A, Izquierdo F. Leishmaniasis tegumentaria americana: Afectación de mucosa. Una revisión en el Hospital Ruíz y Páez. Cd. Bolívar 1983-1992. Tesis de Grado. Universidad de Oriente, Estado Bolívar, Venezuela, 1994.

6. Theodor $\mathrm{O}$. On the clasification of American Phlebotominae. Journal of Medical Entomology 2:171$197,1965$.

7. Young GD, Arias JR. Flebotomos Vectores de Leishmaniasis en las Américas. OPS, cuaderno técnico No. 33. p. 28, 1992.

8. Young GD, Duncan MA. Guide to the identification and geographic distribution of Lutzomyia sand flies in Mexico, The West Indies, Central and South America (Diptera: Psichodidae). Memories of American Entomologic Institute, p. 1-881, 1994. 